\title{
Weight loss interventions for Hispanic women in the USA: a protocol for a systematic review
}

Kristin E. Morrill ${ }^{1 *}$ D, Melissa Lopez-Pentecost ${ }^{2}$, Guadalupe Ballesteros ${ }^{3}$, Jeanne L. Pfander ${ }^{4}$, Melanie D. Hingle ${ }^{1}$, Yann C. Klimentidis ${ }^{5}$ Cynthia A. Thomson ${ }^{6}$ and David O. Garcia ${ }^{6}$

\begin{abstract}
Background: In the U.S., Hispanic women experience a disproportionate rate of obesity and obesity-related chronic diseases. At the same time, Hispanic women remain considerably underrepresented in behavioral weight loss interventions. The purpose of this review is to systematically evaluate the evidence related to the effectiveness of weight loss interventions among Hispanic women in the U.S. This review will identify elements of successful weight loss interventions as well as areas for future research.

Methods/Design: The following databases will be searched to identify all relevant articles (from inception onwards): PubMed, Embase, Scopus, Web of Science (Science Citation Index and Social Sciences Citation Index), PsycINFO, CINAHL, Chicano Database, SPORTDiscus, CAB Abstracts, and Google Scholar. We will include randomized controlled trials and quasi-experimental studies of adult women ( $\geq 18$ years) from Hispanic/Latino background living in the United States. Eligible interventions will target weight-related behaviors (including diet, physical activity, behavior modification and/or their combinations). The review's primary outcome will be weight change (expressed as change in lbs/kg or body mass index (BMI) $\left(\mathrm{kg} / \mathrm{m}^{2}\right)$ ). Three reviewers will independently screen and select data and two will extract data. The methodological quality (or risk of bias) of individual studies will be appraised using the Effective Public Health Practice Project Quality Assessment Tool. A narrative synthesis will describe quality and content of the evidence.
\end{abstract}

Discussion: The aim of this systematic review is to critically examine existing weight loss interventions for Hispanic women in the U.S. and provide quality evidence for the effectiveness of these interventions on weight loss. Further, this review seeks to identify characteristics of effective interventions and suggest future directions for research efforts targeting weight loss in this population. This review will inform the development of future weight loss interventions for this population.

Systematic review registration: PROSPERO CRD42019119094

Keywords: Hispanic, Weight loss, Culturally sensitive, Intervention, Diet, Physical activity

\footnotetext{
*Correspondence: morrill1@email.arizona.edu

'Department of Nutritional Sciences, College of Agriculture \& Life Sciences,

University of Arizona, Tucson, AZ, USA

Full list of author information is available at the end of the article
}

(c) The Author(s). 2019 Open Access This article is distributed under the terms of the Creative Commons Attribution 4.0 International License (http://creativecommons.org/licenses/by/4.0/), which permits unrestricted use, distribution, and reproduction in any medium, provided you give appropriate credit to the original author(s) and the source, provide a link to the Creative Commons license, and indicate if changes were made. The Creative Commons Public Domain Dedication waiver (http://creativecommons.org/publicdomain/zero/1.0/) applies to the data made available in this article, unless otherwise stated. 


\section{Background}

Hispanic women will comprise $25 \%$ of all women in the USA by 2050 if current population growth trends continue $[1,2]$. Hispanic women experience disproportionate rates of obesity with a rate of $50 \%$ compared with $38 \%$ in non-Hispanic White (NHW) women [3]. Obesity is associated with many chronic diseases and health conditions including hypertension, type 2 diabetes, chronic liver disease, heart disease, several cancers, body pain, depression, and overall low quality of life [4-8]. Consequently, Hispanic women are more likely to develop type 2 diabetes and chronic liver disease compared with NHW women and are significantly more likely to die from these conditions $[9,10]$. Efforts to improve modifiable lifestyle behaviors, such as diet and physical activity, to reduce the obesity burden and improve health in Hispanic women, are urgently needed.

Current guidelines for weight management include the use of comprehensive lifestyle interventions consisting of diet, physical activity, and behavior therapy [11]. When effective, these interventions typically result in a weight loss of approximately $18 \mathrm{lbs}$. in a 6-month period or 1-2 lbs. per week [11]. However, long-term maintenance of weight loss remains a challenge as short-term weight loss is typically followed by a regain of $30-50 \%$ of initial body weight over the subsequent $2-3$ years [12, 13]. Within the field of behavioral weight loss interventions, Hispanic women remain considerably underrepresented. A recent systematic review found that Hispanics in general have comprised less than $10 \%$ of participants in behavioral weight loss interventions [14]. One of the largest lifestyle interventions to include Hispanic women, the Diabetes Prevention Program (DPP), was a landmark trial that demonstrated a comprehensive weight loss intervention can reduce incidence and progression of diabetes [15]. By the completion of the study, weight loss in the lifestyle arm was similarly effective in Hispanic women and NHW women with $-5.9 \%$ and $-4.5 \%$ loss of initial body weight after 30 weeks, respectively [16]. The success of the lifestyle arm in inducing short-term weight loss in Hispanic women was likely due, in part, to the range of culturally sensitive materials and strategies implemented to take into consideration the ethnic diversity of study participants (e.g., the use of community lay workers often culturally matched to participants, culturally sensitive cooking materials) [16-18]. Although DPP was a large trial, only $16 \%(n=154)$ of the intensive lifestyle intervention (ILI) group identified as Hispanic women and information including level of acculturation, years in the USA, and country of origin were not collected, thereby limiting generalizability to different sub-populations within the Hispanic community [16]. In the Look AHEAD trial, the longest randomized trial to evaluate the effectiveness of lifestyle modification on weight and cardiovascular-related health outcomes, Hispanic women comprised only $9 \%(n=240)$ of participants in the ILI group and weight loss achieved by year 1 was slowly regained over the next 3 to 7 years, including among Hispanic women $[19,20]$. Additionally, similar to the DPP, the multicomponent lifestyle intervention delivered to participants makes it difficult to ascertain which specific intervention components were effective in inducing the observed weight loss.

Our hypothesis is that Hispanic women are uniquely and importantly different from NHW women when establishing effective programs for weight management. Cultural, social, and economic factors shape the way Hispanic women think and act regarding diet, physical activity, and weight loss. Further, actual and perceived barriers Hispanic women face while attempting to implement lifestyle changes need to be considered as influential elements in developing effective interventions. For example, among Mexican-American women, weight loss efforts may be influenced by a desire for a curvier figure, a desire to feel a part of American society, social hierarchies found within the home, concerns over spending time on themselves, familial pressures, and a lack of social/familial support [21, 22]. Additionally, structural barriers to diet and physical activity, such as the built environment, food deserts, and related issues in regards to access to healthy food may impede efforts to lose weight [23-25]. The process of acculturation has been identified as a factor affecting diet and physical activity behaviors both positively and negatively in individuals immigrating to the USA [26]. For Hispanic women, greater acculturation is positively associated with greater levels of total physical activity throughout the day [27-29] and increased likelihood of engaging in recommended amount of physical activity [30]. While data are limited, westernized dietary acculturation for Hispanic women is characterized as increased intakes of saturated fat, sugar, dessert, and low-fat milk, and decreased intake of corn tortillas, low-fiber bread, and high-fat milk [31]. Given this evidence, the role of sociocultural factors such as level of acculturation and immigrant status remains important to understand and assess while seeking to improve weight loss efforts in Hispanic women. Additionally, woven into these factors are specific values, customs, and perceptions rooted in cultural gender norms that affect the engagement of Hispanic women in weight loss efforts [21, 25]. For example, Hispanic women may perceive a fuller figure to be more "healthy-looking" and desirable and may be deterred from engaging in physical activity by their husbands $[21,25,32,33]$. Hispanic women may find it difficult to adopt healthier eating and cooking habits that would promote weight control for fear of the impact these changes would have on their family [25]. These 
unique barriers faced by Hispanic women attempting to engage in healthy lifestyle behaviors warrant interventions that are tailored to the needs of Hispanic individuals in general but more specifically, the needs of Hispanic women. For this reason, we have opted to focus this review on weight loss interventions that include only Hispanic women.

To date, systematic reviews focused exclusively on U.S. Hispanic women exist for diabetes risk factors management [34], cancer screening [35, 36], factors associated with physical activity [37], and maternal health and breastfeeding [38]. Multiple reviews have examined only physical activity interventions in Hispanic adults [39-41]. In a systematic review by Corona et al., authors examined lifestyle interventions in adult Latinas but did not summarize information related to the effectiveness of the interventions in inducing weight loss, study socio-economic factors, and recruitment variables, and no quality assessment of studies was performed [42]. The current review will extend the literature by providing a comprehensive and rigorous examination of weight loss interventions in Hispanic women and will include an in-depth synthesis of participant characteristics, intervention design, and study quality while using a predetermined measure for intervention success.

The purpose of this systematic review is to characterize previously tested weight loss interventions in Hispanic women in the USA and to identify areas for future research. Additionally, components of successful interventions (those that have achieved clinically meaningful weight loss of $\geq 3 \%$ [11]) will be identified so that future interventions can build on previous findings and ensure meaningful progress is made. A weight loss of $\geq 3 \%$ is associated with clinically meaningful reductions in risk factors for diabetes such as hemoglobin A1c and blood glucose [11] and therefore was chosen as a predetermined measures of intervention effectiveness.

In the current manuscript, the term "Hispanic" is representative of individuals who classify themselves as a person of Mexican, Cuban, South or Central American, Puerto Rican, or other Spanish culture or origin, regardless of race. Importantly, the ongoing Hispanic Community Health Study/Study of Latinos (HCHS/SOL) continues to provide new insights into factors involved in the prevention and treatment of chronic disease among Hispanic/Latino persons from different countries of origin $[43,44]$. We acknowledge the considerable heterogeneity of the term "Hispanic" and will recognize other terms (e.g., Latino/a/x) and/or subgroups (e.g., Mexican American) within our search strategy in efforts to be as inclusive as possible.

\section{Methods and analysis}

\section{Study registration}

This systematic review protocol is being reported in accordance with the reporting guidance in the Preferred
Reporting Items for Systematic Reviews and MetaAnalyses Protocol (PRISMA-P) statement [45]. The PRISMA checklist is provided in Additional file 1. As a systematic review of published studies, ethics approval will not be required. This review has been registered with the International Prospective Register of Systematic Reviews (PROSPERO) (registration number: CRD42019119094; date of registration: April 2, 2019.

\section{Study eligibility criteria}

Studies will be selected according to the criteria outlined in Table 1.

\section{Study participants}

Our decision to focus our review on Hispanic women living in the USA stems from the knowledge that U.S. Hispanic women have unique attitudes, barriers, and facilitators related to weight loss with important sociocultural contextual factors specific to the USA (e.g., acculturation, built environment, high burden of obesity, and related disease) meriting focused examination. Additionally, racial/ethnic- and sex-specific models exploring predictors of weight loss have demonstrated differences in key predictors of weight loss, further justifying the need to independently focus on Hispanic women [20].

\section{Interventions}

Lifestyle interventions $\geq 12$ weeks in duration, a minimum timeframe associated with short-term weight loss [46], targeting diet and/or physical activity to reduce body weight will be included. This will include dietary interventions targeting weight, physical activity interventions targeting weight, and a combination of both with and without behavioral modification. Dietary interventions will be defined as interventions where diet is modified through changes in diet-related behaviors (e.g., portion control, stimulus control, cooking skills). Physical activity interventions will be defined as interventions where physical activity is modified through changes in physical activity-related behaviors (e.g., increasing leisure time physical activity, reducing sedentary behavior). Interventions will not be excluded based on intervention delivery modality, but modality will be tracked as a component that may influence program effects. Additionally, interventions delivered by a range of different interventionists (e.g., students, physicians, community health workers) will be included.

\section{Outcomes and prioritization}

Studies to be included in this review must report weight or body mass index (BMI) $\left(\mathrm{kg} / \mathrm{m}^{2}\right)$ as a primary or secondary outcome. Changes in risk factors for chronic disease (e.g., blood glucose, hemoglobin A1c, and lipids) included as primary or secondary outcomes in eligible 
Table 1 Inclusion and exclusion criteria

\begin{tabular}{|c|c|c|}
\hline PICOS strategy & Inclusion criteria & Exclusion criteria \\
\hline P-Population & $\begin{array}{l}\text { Hispanic women, } 18+\text { years old, } \\
\text { living in the USA }\end{array}$ & $\begin{array}{l}\text { Studies that recruited both men and women (however, } \\
\text { studies that included men and children as a strategy to } \\
\text { engage women will be kept), studies focused on } \\
\text { children and/or adolescents that allowed parents to attend, } \\
\text { patients who are hospitalized or institutionalized, patients } \\
\text { with eating disorders, patients who have recently } \\
\text { undergone bariatric surgery }\end{array}$ \\
\hline I - Intervention & $\begin{array}{l}\text { Lifestyle interventions } \geq 12 \text { weeks in } \\
\text { duration, targeting diet, and/or physical } \\
\text { activity to reduce body weight }\end{array}$ & $\begin{array}{l}\text { Surgical procedures, nonsurgical devices and procedures, } \\
\text { pharmacological treatments, complementary/alternative } \\
\text { treatments, dietary supplements intended for weight loss, } \\
\text { population-focused health promotion campaigns, and } \\
\text { interventions that do not focus on modifying weight, } \\
\text { interventions to prevent excessive weight gain during } \\
\text { pregnancy }\end{array}$ \\
\hline C - Comparison & $\begin{array}{l}\text { For RCTs, wait-list control or usual care } \\
\text { For quasi-experimental, no } \\
\text { comparison required }\end{array}$ & \\
\hline O- Outcome & $\begin{array}{l}\text { Studies reporting objectively measured } \\
\text { weight change (expressed as change in } \\
\left.\text { lbs. or } \mathrm{kg} \text { or BMl }\left(\mathrm{kg} / \mathrm{m}^{2}\right)\right) \text { as a primary or } \\
\text { secondary outcome. }\end{array}$ & Self-reported measures of weight change \\
\hline S - Study design & RCTs and quasi-experimental studies & $\begin{array}{l}\text { Reviews, observational studies (cross sectional, case-control, } \\
\text { and cohort studies), case reports, case series, in vitro studies, } \\
\text { animal studies, secondary analyses of trials, and survey } \\
\text { development studies }\end{array}$ \\
\hline
\end{tabular}

studies will be summarized. Characteristics of interventions that have induced a clinically meaningful weight loss of $\geq 3 \%$ [11] will be summarized.

\section{Data sources and search strategy}

The following databases will be searched by a professional librarian (JLP) for all relevant articles from inception onwards: PubMed, Embase, Scopus, Web of Science (Science Citation Index and Social Sciences Citation Index), PsycINFO, CINAHL, Chicano Database, SPORTDiscus, CAB Abstracts, and Google Scholar. A detailed description of the search strategy for PubMed is included as Additional file 2. Reference lists from all eligible studies will be hand-searched in addition to reference lists of related reviews. The search will be supplemented with suggestions from experts in the field. The search will be limited to publications written in the English language.

\section{Data management}

Results from the search through the electronic databases will be uploaded by JLP into EndNote citation manager software and duplicates removed. The EndNote file will then be uploaded to Covidence systematic review software (Veritas Health Innovation, Melbourne, Australia). KM, MLP, and GB will then independently begin the reviewing process.

\section{Selection process}

Excluding duplicates, articles generated from the search strategy will be divided into three equal sections (A, B, $C)$. Then, three authors (KM, MLP, GB) will independently perform an initial title and abstract review in the following manner: $\mathrm{KM}$ will review $\mathrm{A}+\mathrm{B}$, MLP will review $\mathrm{B}+\mathrm{C}$, and $\mathrm{GB}$ will review $\mathrm{A}+\mathrm{C}$. The three authors will then meet and results will be discussed until a consensus is reached regarding eligible studies. In the next step, remaining articles will be read in full-text format independently by KM and MLP. Discrepancies at these two screening steps will be resolved by DG and the set of articles to be reviewed will be finalized.

\section{Data extraction}

Data from these articles will be extracted independently by KM and MLP and reviewed by GB. Extracted data from the articles will be collected by KM and MLP using a standardized template form developed specifically for this review and cross-referenced by GB for any discrepancies. If data extraction for an eligible study cannot be accomplished due to information inadequately described or missing in the full-text article, KM will contact the publication's corresponding author via email up to three times to request the information. KM and MLP will pilot the standardized template on a subsample of eligible studies and make any appropriate adjustments to the data collection fields as necessary before continuing with the remaining studies. In the case there are multiple 
publications for an eligible study, data will be extracted from each manuscript in order to retrieve all information relevant to this review. Intervention materials, such as educational materials provided to participants, will be requested from trial authors and published as supplementary materials in the final review [47].

Data to be extracted from eligible articles are as follows:

1. Author and year of publication

2. Participant population (age, important eligibility criteria such as weight classification or disease state)

3. Socio-cultural factors (acculturation level and Hispanic subgroup or country of origin, if available)

4. Study design (randomized controlled trial or quasiexperimental design)

5. Recruitment variables (setting, strategy, and effectiveness)

6. Intervention characteristics (duration, study materials, study delivery setting, intervention modality, theoretical frameworks/behavior change techniques, and culturally sensitive strategies implemented)

7. Comparator (if available) and description

8. Study outcome information (weight loss and/or changes in BMI included as primary or secondary outcome) and changes in other risk factors for chronic disease (e.g., blood glucose, hemoglobin A1c, and lipids) included as the secondary study outcomes (if available)

9. Retention and adherence rates

\section{Quality assessment}

Quality in all studies will be independently assessed by KM and MLP using the Effective Public Health Practice Project Quality Assessment Tool (EPHPP) [48]. This standardized tool, developed by the Effective Public Health Practice Project in Canada, was chosen since it can be used to evaluate a range of intervention study designs including RCTs and pre- and post-studies, both of which may be included in the review. The EPHPP evaluates study quality by assessing the following six domains: (1) selection bias; (2) study design; (3) confounders; (4) blinding; (5) data collection; and (6) withdrawals/dropouts. When using this tool, each of these six domains is rated from weak ( 1 point) to strong ( 3 points) and these are averaged to provide a total quality score for the study. Validity and reliability for this tool meets accepted standards [48]. For the current review, all studies to be included in the review will have a copy of the EPHPP attached, and both KM and MLP will independently assess each article. Any discrepancies will be resolved by DG. Because we are not conducting a meta-analysis, there will be no data synthesis, and therefore, no sensitivity analysis will be conducted with the results from this assessment.

\section{Data analysis}

A narrative synthesis of eligible studies will be conducted. This will include qualitatively summarizing all extracted data. Quantitative summaries of extracted data such as the proportion of studies to achieve a clinically meaningful weight loss of $\geq 3 \%$ [11] and frequencies of specific intervention characteristics (e.g., use of theoretical framework, study delivery setting, culturally sensitive strategies implemented) will be reported. KM will synthesize all of the data and this will be reviewed by MLP. Discrepancies will be resolved by DG. These summaries will be used to assess the effectiveness of weight loss interventions for Hispanic women in the USA and address gaps in the existing literature. After synthesizing the data, we will identify areas for future research.

\section{Discussion}

This review will be among the first to provide quality evidence for the effectiveness of weight loss interventions developed specifically for Hispanic women in the USA and identify salient features of effective interventions. This information will be useful to inform the development of future weight loss interventions for this population. Potential limitations include a limited number of eligible studies, studies with small samples sizes, insufficient detail of sample eligibility, and/or intervention strategies and characteristics as well as the exclusion of studies published in non-English languages. Any amendments made to this protocol while conducting the study will be described in PROSPERO and outlined in the final published manuscript. Findings from this review will be widely disseminated through conference presentations and peer-reviewed publications.

\section{Supplementary information}

Supplementary information accompanies this paper at https://doi.org/10. 1186/s13643-019-1213-3.

Additional file 1. PRISMA-P (Preferred Reporting Items for Systematic review and Meta-Analysis Protocols) 2015 checklist: recommended items to address in a systematic review protocol.

Additional file 2. Morrill SR PubMed Search Strategy (07/02/2019).

Abbreviations

BMI: Body mass index; CINAHL: The Cumulative Index to Nursing and Allied Health Literature; DPP: Diabetes Prevention Program; EPHPP: Effective Public Health Practice Project Quality Assessment Tool; PRISMA-P: Preferred Reporting Items for Systematic Reviews and Meta-Analyses Protocol; PROSPERO: International Prospective Register of Systematic Reviews; RCTs: Randomized controlled trials; SCl: Science Citation Index; SSCl: Social Sciences Citation Index 


\section{Acknowledgements}

We wish to thank Ms. Maribeth Slebodnik, BSN, MLS, for her guidance and expertise developing and refining the review's search strategy and Ms. Jean McClelland, MLS, for her valuable advice and guidance.

\section{Authors' contributions}

KEM is the guarantor of this systematic review. KEM, JLP, MDH, YCK, CAT, and DOG contributed to the review's initial conception. KEM and DOG registered the review and contributed to its design. KEM, JLP, and DOG developed the search strategies. JLP, KEM, ML-P, GB, and DOG performed the search and identified studies for review. KEM drafted the protocol manuscript with support from JLP, ML-P, GB, and DOG. All authors critically reviewed and commented on this protocol. All authors read and approved the final manuscript.

\section{Authors' information}

Not applicable

\section{Funding}

This work was supported by the National Needs Graduate Fellowship Program with the United States Department of Agriculture (USDA).

\section{Availability of data and materials}

Data sharing is not applicable to this article as no datasets were generated or analyzed during the current study.

\section{Ethics approval and consent to participate}

Not applicable

\section{Consent for publication}

Not applicable

\section{Competing interests}

The authors declare that they have no competing interests.

\section{Author details}

'Department of Nutritional Sciences, College of Agriculture \& Life Sciences, University of Arizona, Tucson, AZ, USA. ${ }^{2}$ Department of Clinical and Translational Sciences, College of Medicine, University of Arizona, Tucson, AZ, USA. ${ }^{3}$ Department of Physiology, College of Medicine, University of Arizona, Tucson, AZ, USA. ${ }^{4}$ University Libraries, University of Arizona, Tucson, AZ, USA. ${ }^{5}$ Department of Epidemiology and Biostatistics, University of Arizona, Mel and Enid Zuckerman College of Public Health, Tucson, AZ, USA. ${ }^{6}$ Department of Health Promotion Sciences, University of Arizona, Mel and Enid Zuckerman College of Public Health, Tucson, AZ, USA.

\section{Received: 8 July 2019 Accepted: 23 October 2019}

\section{Published online: 01 December 2019}

\section{References}

1. Paz K, Massey KP. Health disparity among Latina women: comparison with non-Latina women. Clin Med Insights Womens Health. 2016;9(Suppl 1):71-4.

2. Ramos BM, Jurkowski J, Gonzalez BA, Lawrence C. Latina women: health and healthcare disparities. Soc Work Public Health. 2010;25(3):258-71.

3. Hales CM, Carroll MD, Fryar CD, Ogden CL. Prevalence of obesity among adults and youth: United States, 2015-2016. NCHS data brief, no 288. Hyattsville: National Center for Health Statistics. 2017

4. Bhaskaran K, Douglas I, Forbes H, dos-Santos-Silva I, Leon DA, Smeeth L. Body-mass index and risk of 22 specific cancers: a population-based cohort study of 5.24 million UK adults. Lancet. 2014:384(9945):755-65.

5. Klein S, Burke LE, Bray GA, Blair S, Allison DB, Pi-Sunyer X, et al. Clinical implications of obesity with specific focus on cardiovascular disease: a statement for professionals from the American Heart Association Council on Nutrition, Physical Activity, and Metabolism: endorsed by the American College of Cardiology Foundation. Circulation. 2004;110(18):2952-67.

6. Pi-Sunyer FX. The obesity epidemic: pathophysiology and consequences of obesity. Obes Res. 2002;10(Suppl 2):97S-104S.

7. Kasen S, Cohen P, Chen H, Must A. Obesity and psychopathology in women: a three decade prospective study. Int J Obes. 2008;32(3):558-66.

8. Luppino FS, de Wit LM, Bouvy PF, Stijnen T, Cuijpers P, Penninx BW, et al. Overweight, obesity, and depression: a systematic review and meta-analysis of longitudinal studies. Arch Gen Psychiatry. 2010;67(3):220-9.
9. Kposowa AJ. Mortality from diabetes by Hispanic groups: evidence from the US National Longitudinal Mortality Study. Int J Popul Res. 2013;2013:12.

10. Ong JP, Pitts A, Younossi ZM. Increased overall mortality and liver-related mortality in non-alcoholic fatty liver disease. J Hepatol. 2008:49(4):608-12.

11. Jensen MD, Ryan DH, Apovian CM, Ard JD, Comuzzie AG, Donato KA, et al. 2013 AHA/ACC/TOS guideline for the management of overweight and obesity in adults: a report of the American College of Cardiology/American Heart Association Task Force on Practice Guidelines and The Obesity Society. J Am Coll Cardiol. 2014;63(25 Pt B):2985-3023.

12. Wadden TA, Neiberg RH, Wing RR, Clark JM, Delahanty LM, Hill JO, et al. Four-year weight losses in the Look AHEAD study: factors associated with long-term success. Obesity (Silver Spring). 2011;19(10):1987-98.

13. Wing RR, Hamman RF, Bray GA, Delahanty L, Edelstein SL, Hill JO, et al. Achieving weight and activity goals among diabetes prevention program lifestyle participants. Obes Res. 2004;12(9):1426-34.

14. Haughton CF, Silfee VJ, Wang ML, Lopez-Cepero AC, Estabrook DP, Frisard C, et al. Racial/ethnic representation in lifestyle weight loss intervention studies in the United States: a systematic review. Prev Med Rep. 2018;9:131-7.

15. Knowler WC, Barrett-Connor E, Fowler SE, Hamman RF, Lachin JM, Walker EA, et al. Reduction in the incidence of type 2 diabetes with lifestyle intervention or metformin. N Engl J Med. 2002;346(6):393-403.

16. West DS, Elaine Prewitt T, Bursac Z, Felix HC. Weight loss of black, white, and Hispanic men and women in the Diabetes Prevention Program. Obesity (Silver Spring). 2008;16(6):1413-20.

17. Hoskin MA, Bray GA, Hattaway K, Khare-Ranade PA, Pomeroy J, Semler LN, et al. Prevention of diabetes through the lifestyle intervention: lessons learned from the diabetes prevention program and outcomes study and its translation to practice. Curr Nutr Rep. 2014;3(4):364-78.

18. Diabetes Prevention Program Research G. The Diabetes Prevention Program (DPP): description of lifestyle intervention. Diabetes Care. 2002; 25(12):2165-71.

19. Wing RR, Espeland MA, Clark JM, Hazuda HP, Knowler WC, Pownall HJ, et al. Association of weight loss maintenance and weight regain on 4-year changes in CVD risk factors: the action for health in diabetes (Look AHEAD) Clinical Trial. Diabetes Care. 2016;39(8):1345-55.

20. West DS, Dutton G, Delahanty LM, Hazuda HP, Rickman AD, Knowler WC, et al. Weight loss experiences of African American, Hispanic, and nonHispanic White men and women with type 2 diabetes: the Look AHEAD Trial. Obesity (Silver Spring). 2019;27(8):1275-84.

21. Lindberg NM, Stevens VJ. Immigration and weight gain: Mexican-American women's perspectives. J Immigr Minor Health. 2011;13(1):155-60.

22. McLaughlin EA, Campos-Melady M, Smith JE, Serier KN, Belon KE, Simmons JD, et al. The role of familism in weight loss treatment for Mexican American women. J Health Psychol. 2017;22(12):1510-23.

23. Tiedje K, Wieland ML, Meiers SJ, Mohamed AA, Formea CM, Ridgeway JL, et al. A focus group study of healthy eating knowledge, practices, and barriers among adult and adolescent immigrants and refugees in the United States. Int J Behav Nutr Phys Act. 2014;11:63.

24. Gerchow L, Tagliaferro B, Squires A, Nicholson J, Savarimuthu SM, Gutnick D, et al. Latina food patterns in the United States: a qualitative metasynthesis. Nurs Res. 2014;63(3):182-93.

25. Larsen BA, Pekmezi D, Marquez B, Benitez TJ, Marcus BH. Physical activity in Latinas: social and environmental influences. Womens Health (Lond). 2013; 9(2):201-10.

26. Winham DM, Palmer SM, Armstrong Florian TL, Shelley MC. Health behaviors among low-income Hispanic and non-Hispanic White women. Am J Health Behav. 2018;42(3):56-68.

27. Slattery ML, Sweeney C, Edwards S, Herrick J, Murtaugh M, Baumgartner K et al. Physical activity patterns and obesity in Hispanic and non-Hispanic white women. Med Sci Sports Exerc. 2006;38(1):33-41.

28. Corral I, Landrine H. Acculturation and ethnic-minority health behavior: a test of the operant model. Health Psychol. 2008;27(6):737-45.

29. Wolin KY, Colditz G, Stoddard AM, Emmons KM, Sorensen G. Acculturation and physical activity in a working class multiethnic population. Prev Med. 2006:42(4):266-72

30. Vermeesch AL, Stommel M. Physical activity and acculturation among U.S Latinas of childbearing age. West J Nurs Res. 2014;36(4):495-511.

31. Batis C, Hernandez-Barrera L, Barquera S, Rivera JA, Popkin BM. Food acculturation drives dietary differences among Mexicans, Mexican Americans, and Non-Hispanic Whites. J Nutr. 2011;141(10):1898-906. 
32. Bautista L, Reininger B, Gay JL, Barroso CS, McCormick JB. Perceived barriers to exercise in Hispanic adults by level of activity. J Phys Act Health. 2011; 8(7):916-25.

33. Ramirez AG, Chalela P, Gallion K, Velez LF. Energy balance feasibility study for Latinas in Texas: a qualitative assessment. Prev Chronic Dis. 2007;4(4):A98.

34. Carolan-Olah M, Duarte-Gardea M, Lechuga J. A systematic review of interventions for Hispanic women with or at risk of Gestational diabetes mellitus (GDM). Sex Reprod Healthc. 2017;13:14-22.

35. Jerome-D'Emilia B. A systematic review of barriers and facilitators to mammography in Hispanic women. J Transcult Nurs. 2015;26(1):73-82

36. Luque JS, Logan A, Soulen G, Armeson KE, Garrett DM, Davila CB, et al. Systematic review of mammography screening educational interventions for Hispanic women in the United States. J Cancer Educ. 2019;34(3):412-22.

37. Tovar M, Walker $\mathrm{JL}$, Rew L. Factors associated with physical Activity in Latina women: a systematic review. West J Nurs Res. 2018;40(2):270-97.

38. Bigman G, Wilkinson AV, Perez A, Homedes N. Acculturation and breastfeeding among Hispanic American women: a systematic review. Matern Child Health J. 2018;22(9):1260-77.

39. Ickes MJ, Sharma M. A systematic review of physical activity interventions in Hispanic adults. J Environ Public Health. 2012;2012:156435.

40. Loya JC. Systematic review of physical activity interventions and Hispanic adults. Hisp Health Care Int. 2018. https://doi.org/10.1177/ 1540415318809427.

41. Joseph RP, Royse KE, Benitez TJ. A systematic review of electronic and mobile health (e- and mHealth) physical activity interventions for African American and Hispanic women. J Phys Act Health. 2019;16(3):230-9.

42. Corona E, Flores YN, Arab L. Trends in evidence-based lifestyle interventions directed at obese and overweight adult Latinos in the US: a systematic review of the literature. J Community Health. 2016;41(3):667-73.

43. Daviglus ML, Talavera GA, Aviles-Santa ML, Allison M, Cai J, Criqui MH, et al. Prevalence of major cardiovascular risk factors and cardiovascular diseases among Hispanic/Latino individuals of diverse backgrounds in the United States. JAMA. 2012;308(17):1775-84.

44. Kaplan RC, Aviles-Santa ML, Parrinello CM, Hanna DB, Jung M, Castaneda SF, et al. Body mass index, sex, and cardiovascular disease risk factors among Hispanic/Latino adults: Hispanic community health study/study of Latinos. J Am Heart Assoc. 2014;3(4):e000923.

45. Shamseer L, Moher D, Clarke M, Ghersi D, Liberati A, Petticrew M, et al. Preferred reporting items for systematic review and meta-analysis protocols (PRISMA-P) 2015: elaboration and explanation. BMJ. 2015;350:97647.

46. Wadden TA, Webb VL, Moran CH, Bailer BA. Lifestyle modification for obesity: new developments in diet, physical activity, and behavior therapy. Circulation. 2012;125(9):1157-70.

47. Hoffmann TC, Oxman AD, loannidis JP, Moher D, Lasserson TJ, Tovey DI, et al. Enhancing the usability of systematic reviews by improving the consideration and description of interventions. BMJ. 2017;358:2998.

48. Thomas BH, Ciliska D, Dobbins M, Micucci S. A process for systematically reviewing the literature: providing the research evidence for public health nursing interventions. Worldviews Evid-Based Nurs. 2004;1(3):176-84.

\section{Publisher's Note}

Springer Nature remains neutral with regard to jurisdictional claims in published maps and institutional affiliations.

Ready to submit your research? Choose BMC and benefit from:
- fast, convenient online submission
- thorough peer review by experienced researchers in your field
- rapid publication on acceptance
- support for research data, including large and complex data types
- gold Open Access which fosters wider collaboration and increased citations
- maximum visibility for your research: over 100M website views per year
At BMC, research is always in progress.
Learn more biomedcentral.com/submissions

